\title{
Performance Evaluation of Routing Protocols in Vehicular Networks
}

\author{
Ameur BENNAOUI, Mustapha GUEZOURI and Mokhtar KECHE \\ Department of Electronics, Faculty of Electrical Engineering, University of Science and Technology (USTO), P.O. Box \\ 1505 - El-m’naouar, Oran, A LGERIA \\ (ameur_bennaoui, mguezouri)@yahoo.fr
}

\begin{abstract}
Vehicular Ad hoc Networks is promising research area to improve traffic safety by developing an intelligent inter-vehicle communication system. Routing of data in a vehicular ad hoc network is a challenging task due to the high dynamics of such a network. This paper presents a performance study of routing protocols in various network environment (Urban, Highway) based on metrics such as throughput, packet delivery ratio and average end-to-end delay. For the implementation purpose we have used network simulator-2 (NS-2) and VanetMobisim.
\end{abstract}

Index Terms - Vehicular Ad hoc Networks, AODV, DSR, NS2, VanetMobiSim

\section{INTRODUCTION}

The VANETs (Vehicular Ad hoc Networks) are a particular case of the mobile networks, in which mobile nodes are vehicles. Vehicular networks are composed of mobile nodes, vehicles equipped with On Board Units (OBU), and stationary nodes called Road Side Units (RSU) attached to infrastructure that will be deployed along the roads. Both OBU and RSU devices have wireless/wired communications capabilities. OBUs communicate with each other and with the RSUs in ad hoc manner [1]. Generally, from the connectivity point of view they could be divided into two main groups [2]:Vehicle-to-Vehicle (V2V) and Vehicle-to-RSU (V2R).

The RSUs can also communicate with each other and with other networks like the internet as shown in Fig1.

A VANET communication platform allows an enormous variety of applications. The main applications of VANET can be categorized as [3]:

(1) Safety-related applications: are the most important kind of applications for VANETs .primarily it provide information and assistance to drivers to avoid such collisions with other vehicles.

(2) Comfort-related applications: to imp rove passenger comfort and traffic efficiency. That could include weather in formation, mobile e-commerce, internet access and other multimedia applications.

(3) Applications for Administration: This service will provide a safe and fast way of information provision from vehicles without the need of stopping them.

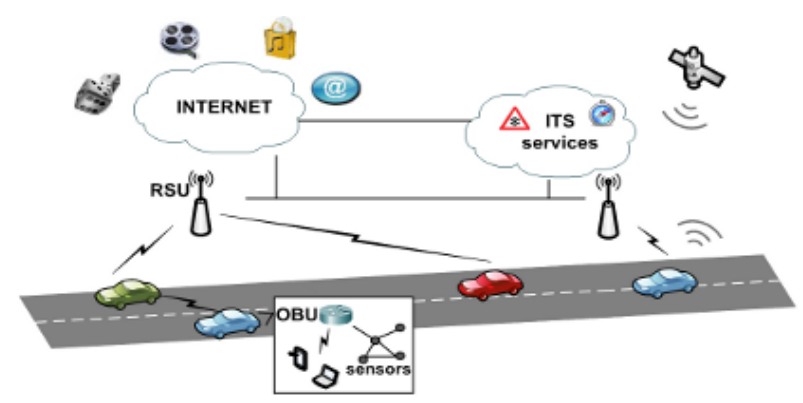

Figure 1. Architecture of Vehicular Ad hoc Networks

The rest of the paper is organized as follows: Section 2 presents of the characteristics of VANET. Section 3 presents the mobile ad hoc routing protocols categories. The simu lation environ ment and performance metrics are described in Section 4 and then the results are presented in Section 5. Finally Section 6 concludes the paper.

\section{II. CARACTERISTICS OF VEHICULAR AD HC NETWORKS}

Vehicular ad hoc network (VANET) has some common properties with MANET, but there are some distinctive characteristics, these characteristics can be summarized as follows [4]:

(1) High mobility: VA NET nodes are characterized by their high relative speed which makes the topology changes very fast.

(2) Predictable and restricted mobility patterns: Un like the random mobility of MANET, VANET node movements are governed by restricted rules (traffic flow theory rules), which make them predictable at least on the short run.

(3) No power constraints: Each vehicle is equipped with a battery that is used as an infinite power supply for all communications and computation tasks.

(4) Localization: Vehicles can use the Global Positioning System (GPS) to identify their locations with high accuracy.

(5) Hard delay constraints: Safety messages are the main goal of VANETs. Therefore, safety messages should be given high priority and must be delivered on time. 


\section{OVERVIEW OF ROUTING PROTOCOLS}

Routing protocols for Mobile ad hoc networks can be classified into two ma in categories(Fig 2):

(1) Proactive routing protocols: each node continuously maintains up-to-date routes to every other node in the network. Routing information is periodically transmitted throughout the network in order to maintain routing table consistency [5].

(2) Reactive routing protocols: In contrast to proactive approach, a node initiates a route discovery throughout the network, only when it wants to send packets to its destination. For this purpose, a node initiates a route discovery process through the network. This process is completed once a route is determined or all possible permutations have been examined. Once a route has been established, it is maintained by a route maintenance process until either the destination becomes inaccessible along every path from the source or until the route is no longer desired [5].

Here we discuss the two reactive type of protocols AODV and DSR:
DSR: Dynamic Source Routing (DSR) protocol is an entirely on-demand ad hoc network routing protocol composed of two parts: Route Discovery and Route Maintenance. In DSR, when a node has a packet to send to some destination and does not currently have a route to that destination in its Route Cache, the node initiates Route Discovery to find a route; this node is known as the initiator of the Route Discovery, and the destination of the packet is known as the Discovery's target [6].

AODV: The Ad Hoc On-demand Distance Vector Routing (AODV) protocol is a reactive unicast routing protocol for mobile ad hoc networks. As a reactive routing protocol, AODV only needs to maintain the routing information about the active paths. In AODV, the routing information is maintained in the routing tables at all the nodes. Every mobile node keeps a next hop routing table, which contains the destinations to which it currently has a route. A routing table entry expires if it has not been used or reactivated for a pre-specified expiration time [6].

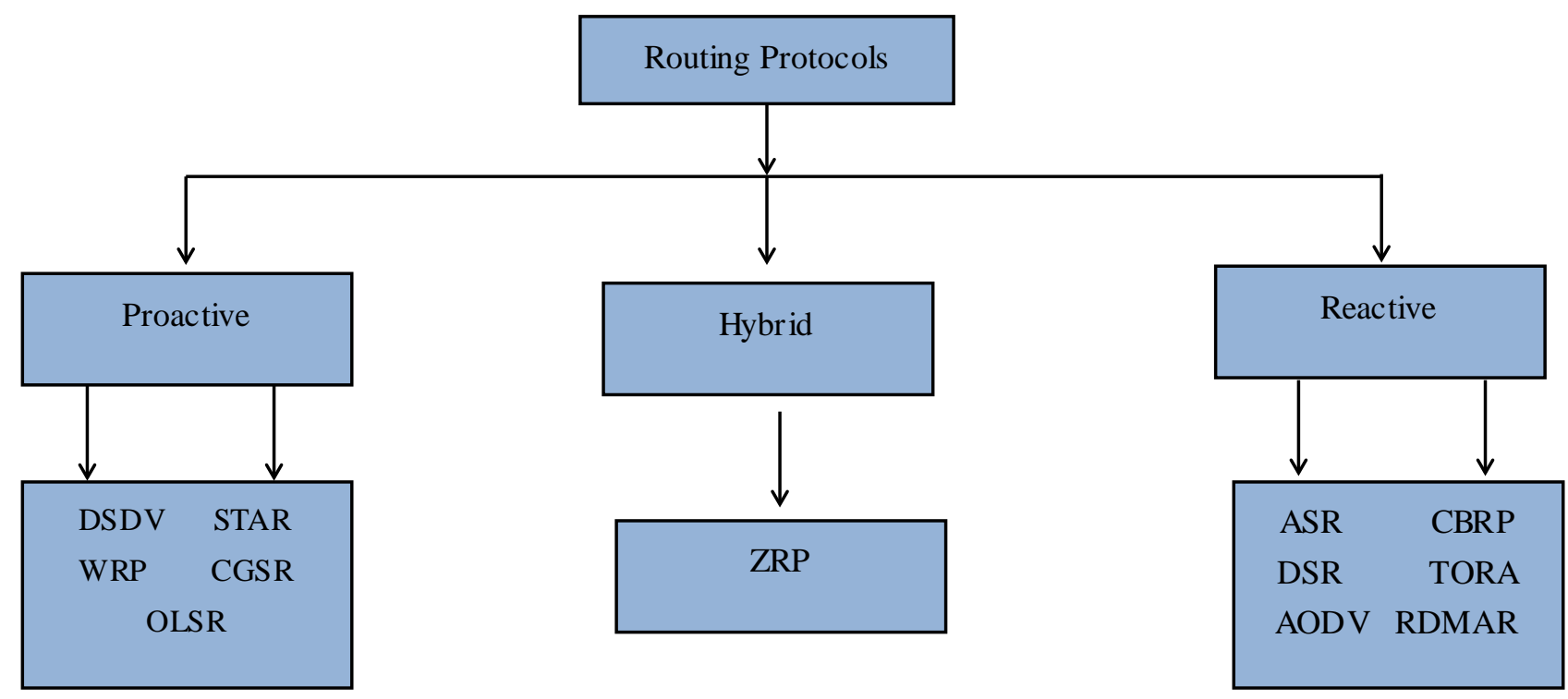

Figure.2 Routing protocols for Mobile ad hoc networks

Unlike DSR routing protocol, AODV determines a route to a destination only when a node wants to send a packet to a destination. Routes are maintained as long as they are needed by the source.

\section{SIMULATION ENVIRONMENT}

In this study , we used a simulator tool based on the combination of the road traffic simulator VanetMobiSim [7] and the network simulator Ns-2 [8].the VanetMobiSim is specific for VANETs and an open source platform; it supports both macro-mobility level (flows of vehicles, planned routes..) and micro-mobility level (acceleration/deceleration, car following each other,..) specification. It used to generate a mobility trace which can be immediately used by a simulation tool such as ns-2 to simulate realistic vehicle movements [9].

In our study we want to check the performance of different routing protocols in different network environments of VANET. For this purpose we built two different network scenarios and named them as highway and urban:

\section{(1) Urban scenario}

A $500 \times 500 \mathrm{~m}^{2}$ dimension has been selected (correspond an area from Tiger /line database)[10], including 10 traffic lights with step of 20 seconds. In addition, the scenarios have been configured with single-lane as well as multi-lane lanes and differentiate two traffic flows. Regarding nodes, several speeds have been selected as well as level of congestion. In addition, 
node's motion is enhanced with Intelligent Driving Model (IDM) [11], which incorporates intersection management and lane changing (Fig 3).

\section{(2) Highway scenario}

The configuration of the scenario is quite similar to the urban scenario except that now dimension is 2000x500 $\mathrm{m}^{2}$ and no traffic lights have been incorporated. In addition, highway has been implemented with two lanes for each traffic flow.

The Table 1 in below summarizes the features of each scenario

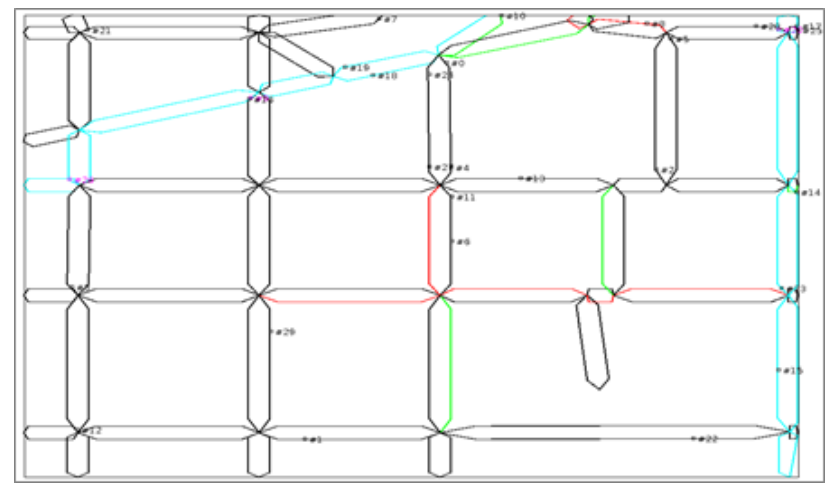

Figure.3. Urban Scenario (from VanetMobSim)

Table 1: Scenario features in VanetMobiSim.

\begin{tabular}{|l|c|c|}
\hline & Urban Scenario & Highway Scenario \\
\hline $\begin{array}{l}\text { Dimension } \\
\left(\mathrm{m}^{2}\right)\end{array}$ & $500 * 500$ & $2000 * 500$ \\
\hline Node Number & $30,60,100,160$ & $30,60,100,160$ \\
\hline Traffic lights & 6 & $/$ \\
\hline $\begin{array}{c}\text { Lane } \\
\text { Configur ation }\end{array}$ & 02 Lanes & 04 lanes \\
\hline Node Speed & $30 \mathrm{~km} / \mathrm{h}$ & $120 \mathrm{~km} / \mathrm{h}$ \\
\hline Driver Model Driven & $\begin{array}{l}\text { Intelligent Driven } \\
\text { Model (IDM-LC). }\end{array}$ & $\begin{array}{l}\text { Intelligent } \\
\text { Model (IDM-LC) }\end{array}$ \\
\hline
\end{tabular}

In The simulations, VanetMobiSim is used to generate the movement file for different number of vehicles with different speeds. This movement trace file is used by
NS-2, a packet-level simulator specifically designed for ad-hoc networks (Fig 4).

The simulation parameters which have been considered for doing the performance comparis on of two o routing protocols is given below in Table- 2

Table 2: Simulation Settings for NS2.

\begin{tabular}{|l|c|c|}
\hline & $\begin{array}{c}\text { Urban } \\
\text { Scenario }\end{array}$ & $\begin{array}{c}\text { Highway } \\
\text { Scenario }\end{array}$ \\
\hline Simulation Time & $600 \mathrm{~s}$ & $600 \mathrm{~s}$ \\
\hline Number of Nodes & $30,60,100,160$ & $30,60,100,160$ \\
\hline Traffic Agent & CBR/UDP & CBR/UDP \\
\hline Packet length & 512 bytes & 512 bytes \\
\hline Data rate & $200 \mathrm{kbps}$ & $200 \mathrm{kbps}$ \\
\hline MAC Protocol & IEEE $802.11 \mathrm{p}$ & IEEE $802.11 \mathrm{p}$ \\
\hline Routing Protocol & AODV,DSR & AODV,DSR \\
\hline
\end{tabular}

\section{RESULTS AND ANALYSES}

We selected AODV and DSR routing protocols to evaluate their performance in the two scenario of VANET (Urban and Highway environment) in terms of different performance metrics [12]:

-Packet Delivery Ratio (PDR): is the ratio between the numbers of packets received by the application layer of destination nodes to the number of packets sent by the application layer of source nodes.

-Average End to End Delay (E2E): Th is is the average time delay for data packets from the source node to the destination node..

-Throughput (TH): It is the average rate of successful message delivery over a time interval.

\section{(1) Urban scenario}

In Figure 5, the packet delivery ratio has been evaluated for DSR and AODV protocols in the urban environment. The number of vehicles in this scenario has been varied from 30 to 160 . The PDR values, for AODV and DSR protocols, range from $51 \%$ to $43 \%$ and $31 \%$ to $8 \%$ respectively. Clearly, higher vehicle densities on an urban significantly decrease the packet delivery ratio for the both protocols

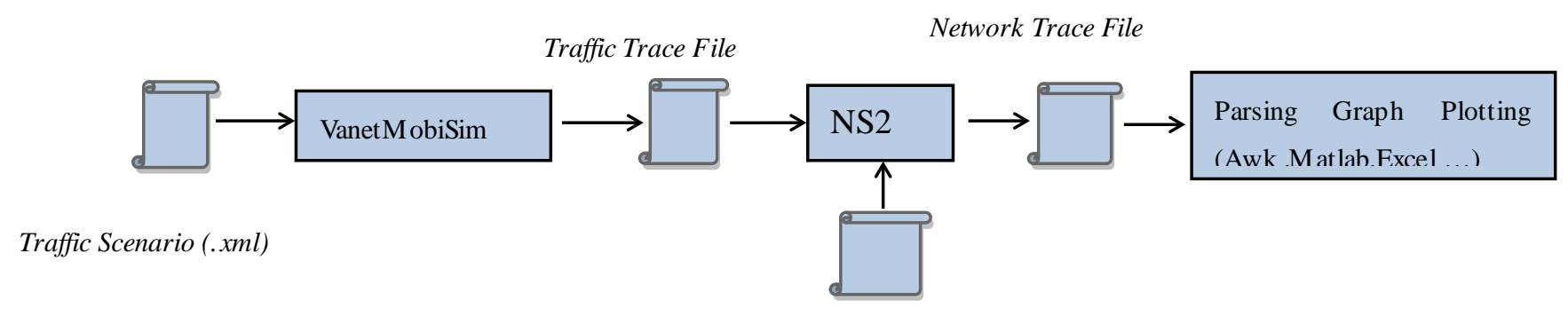

Network scenario

(.tcl)

Figure 4. VANET simulation based on coupling VanetMobiSim and NS2 


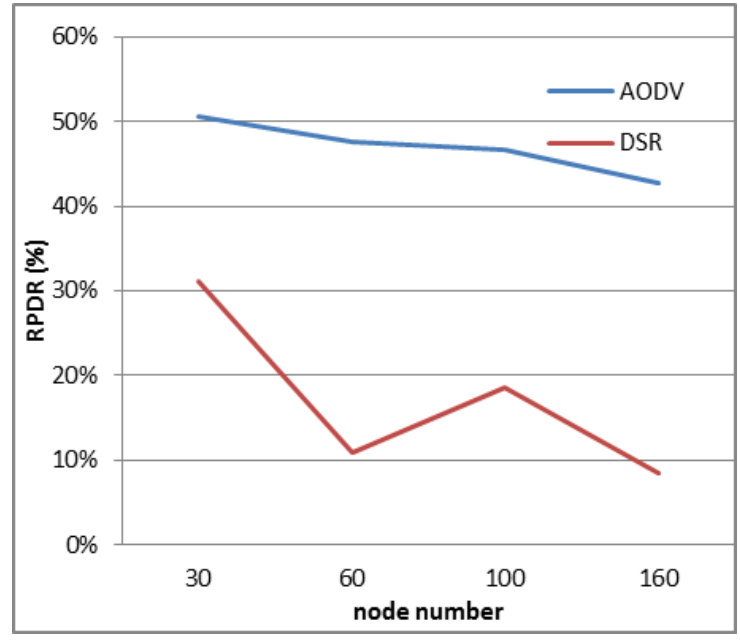

Figure5. Packet Delivery Ratio versus numberof vehicles (Urban scenario)

In Figure 6, the end to end delay has been evaluated for DSR and AODV protocols in the urban environment. The number of vehicles in this

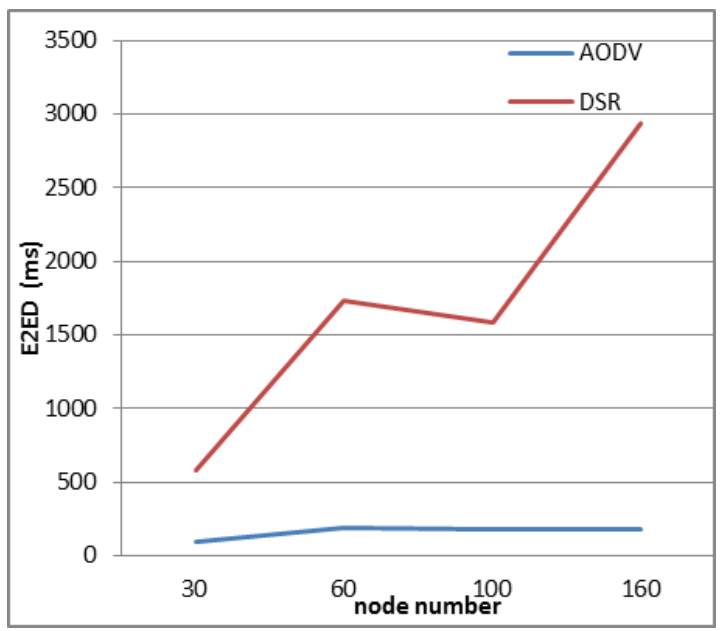

Figure6. End to End Delay versus number of vehicles (Urban scenario)

scenario has been varied from 30 to 160 . We show that the increase in vehicles number in the urban environment has a greater effect on DSR than AODV.

The observation is that the AODV protocol outperforms DSR in term of End to End delay.

Figure 7 and Figure 8 illustrate the comparison of throughput for AODV and DSR protocols in the urban environment. The number of vehicles in this scenario has been 30 and 160. We show, AODV exhibited the highest throughput compared to DSR, and, higher vehicle densities on an urban significantly decrease the Throughput for the both protocols.

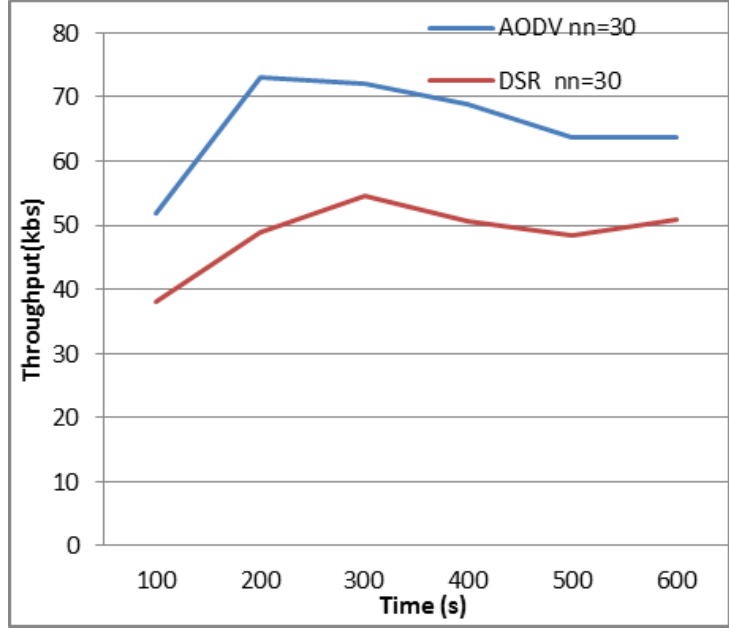

Figure7. Throughput versus Time (Vehicles number $=30$, Urban scenario)

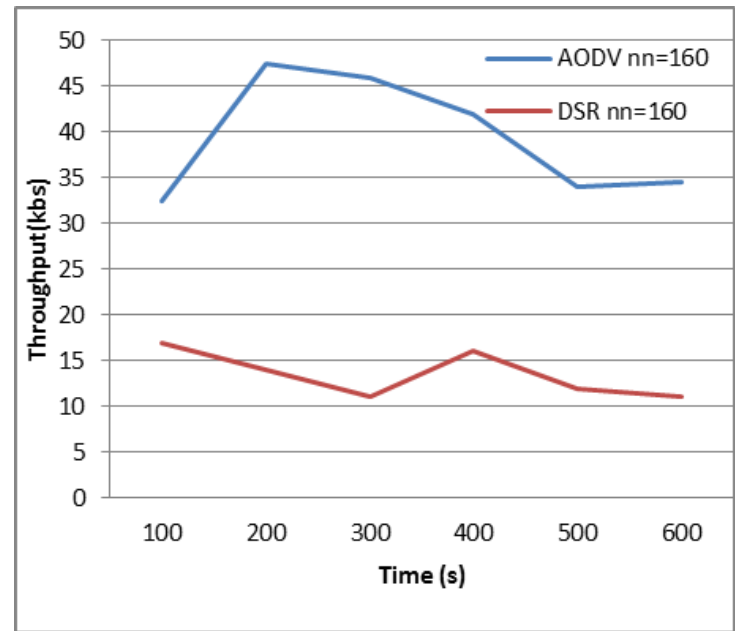

Figure 8. Throughput versus Time (Vehicles number $=160$, Urban scenario)

\section{(2) Highway scenario}

In Figure 9, the packet delivery ratio has been evaluated for DSR and AODV protocols in the Highway environment. The number of vehicles in this scenario has been varied from 30 to 160 . The PDR values, for AODV and DSR protocols, range from $39 \%$ to $37 \%$ and $38 \%$ to $13 \%$ respectively. The observation is that the DSR and AODV protocol gives approximately same PDR values when a low number of vehicles (30).With large numbers of vehicles, AODV starts outperforming DSR for Highway scenario. 


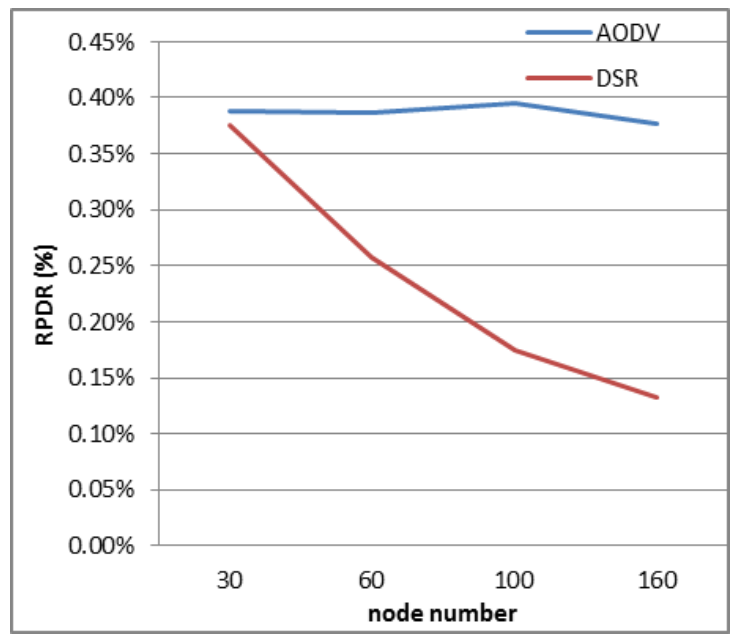

Figure 9. Packet Delivery Ratio versus number of vehicles (Highway scenario)

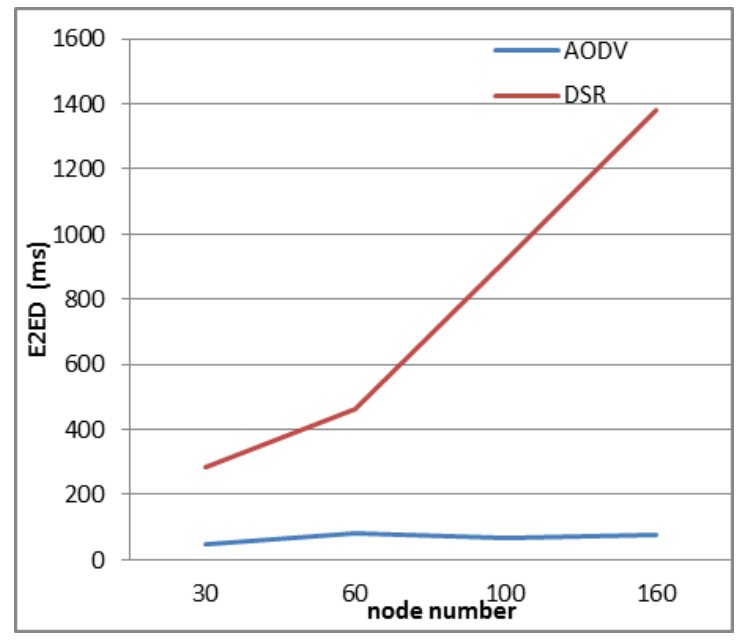

Figure 10. End to End Delay versus number of vehicles (Highway scenario)

In Figure 10, the end to end delay has been evaluated for DSR and AODV protocols in the Highway environment. The number of vehicles in this scenario has been varied from 30 to 160 . The observation is that the AODV protocol outperforms DSR in term of End to End delay.

Figure 11 and Figure 12 illustrate the comparis on of throughput for AODV and DSR protocols in the urban environment. The number of vehicles in this scenario has been 30 and 160 . We show, when the number of vehicles is low (30), the performance of DSR and AODV is similar. With large numbers of vehicles, AODV starts outperforming DSR for Highway scenarios.

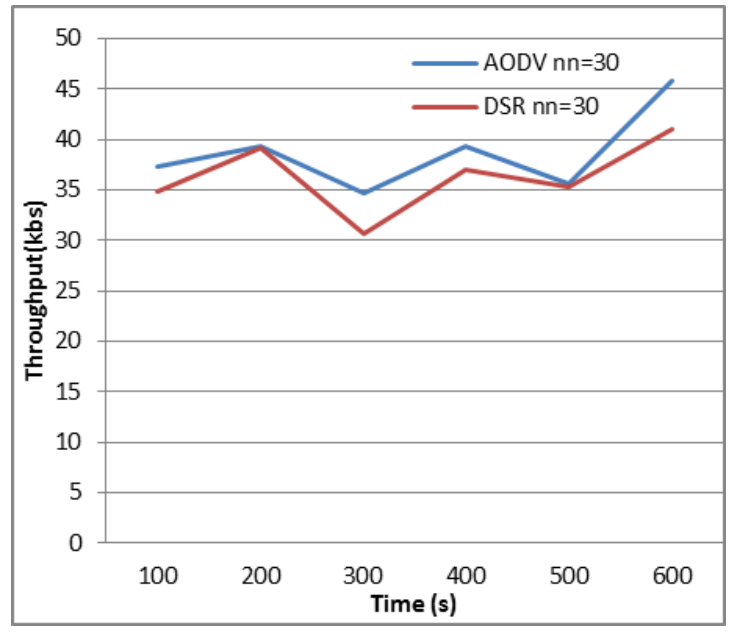

Figure 11. Throughput versus a Time (Vehicles number $=30$, Highway scenario)

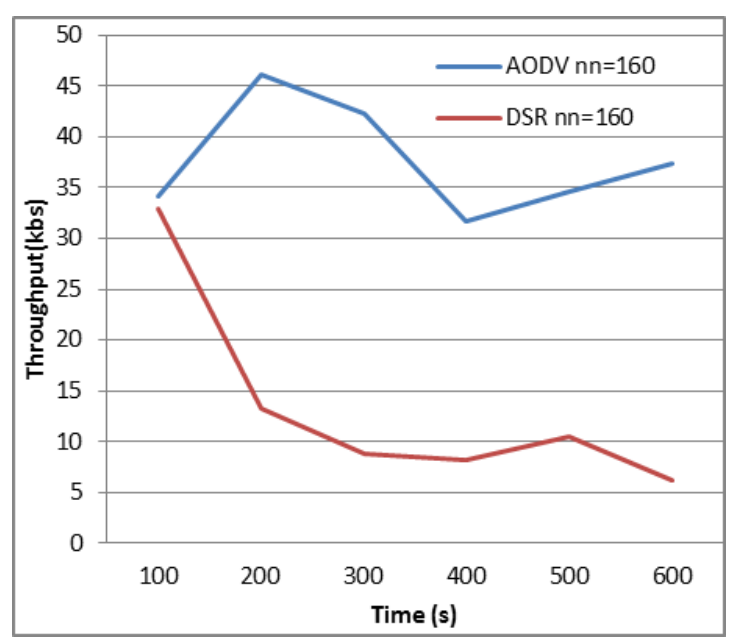

Figure12. Throughput versus Time (Vehicles number $=160$, Highway scenario)

\section{CONCLUSIONS}

In this work, we have presented the results of performance study two routing protocols (AODV and DSR) in two vehicular networks environ ments (Highway, Urban) in term of: packet delivery ratio, average end to end delay and throughput. .In the two scenarios (urban and highway), the results show that: AODV outperforms DSR completely in both scenarios; also that both protocols are sensitive to network size (vehicles' number) and vehicles' speed.

\section{REFERENCES}

[1] H. Hartenstein and K. Laberteaux ,VANET Vehicular Applications and Inter-Networking Technologies. Chapter 7, Wiley, ISBN 978-0-470-74056-9, pp. 219-272, 2010.

[2] Yogesh Suryawanshi , Avichal Kapur ,Analysis of Routing Protocol in Vehicular Ad hoc Network. International Journal of Advanced Engineering 
Sciences and Technologies, Vol no. 8, issue no. 2, $281-285$.

[3] Roger Calzada. ,Performance evaluation of realistic scenarios for vehicular Ad Hoc networks with VanetMobiSim and ns2. Proyecto final de carrera, UPC, 2011.

[4] Zaydoun Yahya Rawashdeh and Syed Masud Mahmud. Communications in Vehicular Ad Hoc Networks, Mobile Ad-Hoc Networks: Applications, Xin Wang (Ed.), ISBN: 978-953-307-416-0, InTech, DOI: 10.5772/13399, 2011.

[5] Anuj K., Gupta Harsh Sadawarti and Anil K. Verma , Performance analysis of AODV, DSR \& TORA Routing Protocols ,IACSIT International Journal of Engineering and Technology, Vol.2, No.2, April 2010 ISSN: 1793-8236.

[6] Yun-Wei Lin, Yuh-Shyan Chen and Sing-Ling Lee. Routing in Vehicular Ad Hoc Networks: A Survey and Future Perspectives,Journal of Information Science and Engineering, 2010.

[7] http://vanet.eurecom.fr.

[8] NS-2 Network simulator http://www.isi.edu/nsnam/ns.

[9] CANU Research Group (Stuttgart University) http://canu.informatik.uni-stuttgart.de/mobisim/.

[10] TIGER(Topologically Integrated GEographic Encoding and. Referencing), "http://www.census.gov/geo/www/tiger/.

[11] B. Ramakrishnan, Dr. R. S. Rajesh, R. S. Shaji ,Analysis of Routing Protocols for Highway Model without Using Roadside Unit and Cluster. International Journal of Scientific \& Engineering Research, Volume 2, Issue 1, January-2011.

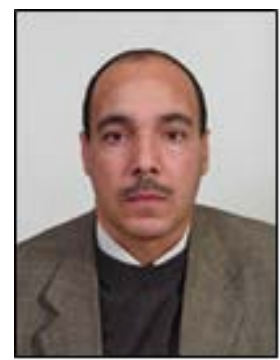

M.GUEZOURI was born in Algeria in. He received the engineer degree from the national Algerian institute of Telecommunications (ITOran) in 1987. He received the Master and $\mathrm{PhD}$ degrees in electrical engineering in 1990 and 2007, respectively from the Science and Technology University in Oran. Since 1990, he has been a Research Assistant at Signal and processing Laboratory, University in Oran.

He is currently an Assistant Professor of electrical and computer engineering at the University in Oran, Algeria. His current research interests include Signal processing, neural network, mesh networks, VANET networks and computer security.

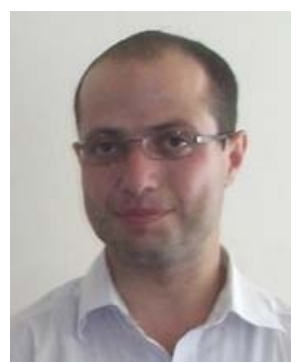

A.BENNAOUI received the engineer degree in telecom systems and the master degree in ICT from INTTIC, Oran, Algeria in 2006 and 2010 respectively. $\mathrm{He}$ is currently a Research Assistant of electrical and computer engineering at the University of Constantine -2-,

Algeria.

His current research interests artificial immune systems, bio-inspired computation and VANET networks. 